\title{
OPTIMAL RESULTS ON RECOGNIZABILITY FOR INFINITE TIME REGISTER MACHINES
}

\author{
MERLIN CARL
}

\author{
Fachbereich Mathematik und Statistik, Universität Konstanz \\ 78457 Konstanz ,Germany \\ merlin.carl@uni-konstanz.de
}

\begin{abstract}
Exploring further the properties of ITRM-recognizable reals started in $[\mathrm{Ca}]$, we provide a detailed analysis of recognizable reals and their distribution in Gödels constructible universe $L$. In particular, we show that new unrecognizables are generated at every index $\gamma \geq \omega_{\omega}^{C K}$. We give a machine-independent characterization of recognizability by proving that a real $r$ is recognizable iff it is $\Sigma_{1}$-definable over $L_{\omega_{\omega}^{C K, r}}$ and that $r \in L_{\omega_{\omega}^{C K, r}}$ for every recognizable real $r$ and show that either every or no $r$ with $r \in L_{\omega_{\omega}^{C K, r}}$ generated over an index stage $L_{\gamma}$ is recognizable. Finally, the techniques developed along the way allow us to prove that the halting number for ITRMs is recognizable and that the set of ITRM-computable reals is not ITRM-decidable.
\end{abstract}

Keywords: Infinite Time Computability, Generalized Computability Infinite Time Register Machines, Recognizable Reals

Classification: 03D65 (Higher-type and set recursion theory), 03D60 Computability and recursion theory on ordinals, admissible sets, etc., 03Exx (Set theory), 03E45 (Inner models, including constructibility, ordinal definability, and core models)

\section{INTRODUCTION}

Infinite Time Register Machines (ITRM's) were introduced by Peter Koepke and Russell Miller in [KoMi] and provide a transfinite analogue of classical register machines in much the same way as the Infinite Time Turing Machines (ITTMs) introduced in [HaLe] generalize classical Turing machines. We give a brief sketch of their behaviour here; detailed definitions can be found in [KoMi] and [ITRM]. These papers also contain most of the results we use in this paper.

Classical register machines, as e.g. described in $[\mathrm{Cu}]$, have as their 'hardware' finitely many registers, each of which can store a single natural number. A program for a register machine consists of finitely many numbered lines, each of which contains a single command, where the available commands are incrementing a register content by 1 , setting a register content to 0 , copying the content from one register to another, 
the oracle call (which replaces the content $i$ of some register $R$ with the $i$ th bit of an oracle $x \subseteq \omega$ ) and a conditional jump which proceeds to a fixed program line when two registers have the same content and otherwise proceeds with the next program line. When the machine arrives at a line index which is not part of the program, the machine halts.

To generalize these machines to the transfinite, we keep the 'hardware' - finitely many registers, each of which stores a single natural number - as well as the 'software', i.e. the notion of program. What is changed is the 'working time': While the working time of a halting classical register machine just a natural number, ITRMs run along arbitrary ordinals. To make sense of this, we need to explain what state the machine is supposed to assume at a limit ordinal. At successor ordinals, we define the computation in the same way as for classical register machines. If $\lambda$ is a limit ordinal, we set the content $R_{i \lambda}$ of the $i$-th register $R_{i}$ at time $\lambda$ to $\liminf \operatorname{in}_{\iota<\lambda} R_{i \iota}$ iff this limit $<\omega$, and otherwise to 0 . Likewise, the active program line $Z_{\lambda}$ to be carried out in the $\lambda$ th step is $\lim \inf _{\iota<\lambda} Z_{\iota}$, (where the limit is always finite as the set of lines is finite).

Definition 1. $x \subseteq \omega$ is ITRM-computable in the oracle $y \subseteq \omega$ iff there exists an ITRM-program $P$ such that, for $i \in \omega, P$ with oracle $y$ stops whatever natural number $j$ is in its first register at the start of the computation and returns 1 iff $j \in x$ and otherwise returns 0 . A real ITRM-computable in the empty oracle is simply called ITRMcomputable.

The notion of the recognizability of a real was introduced by Hamkins and Lewis in [HaLe] for Infinite Time Turing machines. The adaption to ITRMs is straightforward:

Definition 2. Let $r \subseteq \omega$. Then $r$ is recognizable iff there is an ITRMprogram $P$ such that $P^{x}$ stops with output 1 iff $x=r$ and otherwise stops with output 0 .

In the last section of [ITRM], we showed that there are recognizable reals that are not computable. This is analogous to the lost melody theorem for infinite time Turing machines (ITTMs) demonstrated in [HaLe]. The example given there was a $<_{L}$-minimal real coding an $\in$-minimal $L_{\alpha}$ modelling $Z F^{-}$(see below). Here, we will give a much more natural example by showing that the real coding the halting problem for ITRMs is recognizable. After that, in $[\mathrm{Ca}]$, we obtained some more results on ITRM-recognizable reals and their relation to Gödel's constructible universe $L$. In particular, we showed that all recognizable reals are constructible, but that the recognizable reals do not form an initial segment of the constructible reals in the canonical well-ordering $<_{L}$ of $L$. In fact, there are quite large gaps: If 
$\sigma$ is the supremum of the ordinals indexing levels of the $L$-hierarchy at which new recognizable reals appear and $\alpha<\sigma$, then there is a $<_{L}$-interval of length $>\alpha$ in the constructible reals without a recognizable element, while there are recognizable reals that are $<_{L}$-greater than all elements of that interval. Here, our goal is to give a more precise picture of ITRM-recognizability and their distribution among the constructible reals. For example, it is easy to see that all computable reals are recognizable, so that, by the theorem cited above, all reals in $L_{\omega_{\omega}^{C K}}$ are recognizable. Is there any $\alpha>\omega_{\omega}^{C K}$ such that the reals in $L_{\alpha}$ are still all recognizable? It turns out that this is not the case: Whenever $\gamma>\omega_{\omega}^{C K}$ is such that $L_{\gamma+1}-L_{\gamma}$ contains a real number at all, it also contains a non-recognizable real. Furthermore, we give a machine-independent, purely set-theoretical characterization of ITRM-recognizability and lower estimates on the length of gaps in the recognizables and some information on ordinals starting them. The techniques used in these results will then be applied to give a characterization of ITRM-decidable sets of reals and show that the set of ITRM-computable reals is not decidable.

Most of our notation is standard. $Z F^{-}$is $Z F$ set theory without the power set axiom in the version described in [GHJ]. For an ITRMprogram $P, P^{x}(i) \downarrow=j$ means that the program $P$ with oracle $x$ with initial input $i$ in its first register stops with output $j$ in register 1 . On is the class of ordinals. Unless stated otherwise, small greek letters refer to ordinals. For, $\iota \in O n$, we denote by $\omega_{\iota}^{C K}$ the smallest admissible ordinal greater than $\omega_{\gamma}^{C K}$ when $\iota=\gamma+1$ and $\sup \left\{\omega_{\gamma}^{C K} \mid \gamma<\iota\right\}$ when $\iota$ is a limit ordinal. When we consider admissible ordinals relative to a real $x$, we write $\omega_{\iota}^{C K, x}$. For $X \subseteq L_{\alpha}, \Sigma_{1}^{L_{\alpha}}\{X\}$ denotes the $\Sigma_{1^{-}}$ Skolem hull of $X$ in $L_{\alpha}$ and $\Sigma_{\omega}^{L_{\alpha}}\{X\}$ denotes the elementary hull of $X$ in $L_{\alpha}$. When $H$ is a $\Sigma_{1}$-substructure of some $L_{\alpha}$, then $\pi: H \equiv L_{\gamma}$ and $\pi: H \rightarrow$ coll $L_{\gamma}$ denote the Mostowski collapse of $H$ to $L_{\gamma}$ with isomorphism $\pi$. Throughout the paper, $p: \omega \times \omega \rightarrow \omega$ denotes the usual bijection between $\omega \times \omega$ and $\omega$. At various places, we will code countable $\in$-structures by real numbers. This works as follows: Let $(M, \in)$ be countable, and let $f: \omega \rightarrow M$ be a bijection. Then the real coding $(M, \in)$ according to $f$ is $\left\{p(i, j) \mid[f(i) \in f(j)]^{M}\right\}$. We say that $x$ codes $(M, \in)$ iff there is a function $f$ such that $x$ codes $(M, \in)$ according to $f$. If $(M, \in) \in L, c c(M)$ denotes the $<_{L}$-smallest real coding $(M, \in)$.

\section{ITRMS}

We give here some basic results on ITRMs and admissible set theory which are relevant for our further development. 
Theorem 3. Let $\mathbb{P}_{n}$ denote the set of ITRM-programs using at most $n$ registers, and let $\left(P_{i, n} \mid i \in \omega\right)$ enumerate $\mathbb{P}_{n}$ in some natural way. Then the bounded halting problem $H_{n}^{x}:=\left\{i \in \omega \mid P_{i, n}^{x} \downarrow\right\}$ is computable uniformly in the oracle $x$ by an ITRM-program.

Furthermore, if $P \in \mathbb{P}_{n}$ and $P_{x} \downarrow$, then $P_{x}$ halts in less than $\omega_{n+1}^{C K, x}$ many steps. Consequently, if $P$ is a halting ITRM-program, then $P^{x}$ stops in less than $\omega_{\omega}^{C K, x}$ many steps.

Proof. The corresponding results from [KoMi] easily relativize.

Definition 4. $X \subseteq \mathfrak{P}(\omega)$ is ITRM-decidable iff there exists an ITRMprogram $P$ such that $P^{x} \downarrow=1$ iff $x \in X$ and $P^{x} \downarrow=0$, otherwise.

Corollary 5. Let $A$ be an ITRM-decidable set of reals and let $P$ be an ITRM-program. Then $M_{1}:=\left\{x \mid P^{x} \downarrow\right\}, M_{2}:=\left\{x \mid \forall i \in \omega P^{x}(i) \downarrow\right\}$ and $M_{3}:=\left\{x \mid P^{x}\right.$ computes and element of $\left.A\right\}$ are decidable.

Proof. The decidability of $M_{1}$ and $M_{2}$ is immediate from Theorem 3. For $M_{3}$, let $Q$ be a program for deciding $A$. To decide $M_{2}$, proceed as follows: Given some real $x$ in the oracle, first check, whether $x \in M_{2}$. If not, then $x \notin A$. Otherwise, $P^{x}$ computes some real $y$, and we can use $Q$ to decide whether $y \in A$.

Lemma 6. Let $\left(\phi_{i} \mid i \in \omega\right)$ be a natural enumeration of the $\in$-formulas. There is an ITRM-program $P$ such that, for all $x \subseteq \omega, i \in \omega$, $\vec{v}=\left(v_{1}, \ldots, v_{n}\right)$ a finite sequence of natural numbers of the appropriate length coded by a natural number $\bar{v}, P^{x}(i, \vec{v}) \downarrow=1$ iff $x$ codes some $L_{\alpha}$ such that $\phi_{i}\left(x_{1}, \ldots, x_{n}\right)$ holds in $L_{\alpha}$, where $x_{1}, \ldots, x_{n}$ are the elements coded by $v_{1}, \ldots, v_{n}$, respectively, and otherwise $P^{x}(i, \vec{v}) \downarrow=0$. The same holds with a recursive set $S$ of formulas instead of one single formula $\phi$, where $i$ is then a code for a Turing program enumerating $S$.

Proof. This is Corollary 13 of [Ca].

Definition 7. An ordinal $\alpha$ is called $\Sigma_{1}$-fixed iff there exists a $\Sigma_{1^{-}}$ statement $\phi$ such that $\alpha$ is minimal with the property that $L_{\alpha} \models \phi$. Let $\sigma$ denote the supremum of the $\Sigma_{1}$-fixed ordinals.

Theorem 8. Denote by $R E C O G$ the set of recognizable reals. Then $R E C O G \subseteq L_{\sigma}$. Furthermore, for each $\gamma<\sigma$, there exists $\alpha<\sigma$ such that $[\alpha, \alpha+\gamma]$ contains unboundedly many indices, but $\left(L_{\alpha+\gamma}-L_{\alpha}\right) \cap$ $R E C O G=\emptyset$.

Proof. See [Ca].

Theorem 9. Let $x, y \subseteq \omega$. Then $x$ is ITRM-computable in the oracle $y$ iff $x \in L_{\omega_{\omega}^{C K, y}}[y]$.

Proof. This is a straightforward relativization of the main result of [KoMi]. 
Lemma 10. Let $A \neq \emptyset$ be an ITRM-decidable set of reals such that $a \in L_{\omega_{\omega}}^{C K, a}$ for all $a \in A$. Then the $<_{L^{-}}$-minimal element of $A$ is recognizable.

Proof. Let $a$ be the $<_{L}$-minimal element of such an $A$. By Theorem 9, there is an ITRM-program $P$ such that $P^{a}$ computes a code for some $L$-level $L_{\alpha}$ containing $a$. Let $Q_{A}$ be an ITRM-program deciding $A$. Now $a$ can be recognized as follows: Given some $x \subseteq \omega$ in the oracle, first check whether $P^{x}(i) \downarrow$ for all $i \in \omega$, using a halting problem solver for $P$ which exists by Theorem 3. If not, then $x \neq a$. Otherwise, test whether $P^{x}$ computes a code $c$ for an $L$-level containing $x$. This can be done using Lemma 5 and Lemma 6 . If not, then $x \neq a$. Otherwise, test whether $Q_{A}(x) \downarrow=1$. If not, then $x \notin A$, so $x \neq a$. Otherwise, use $c$ to search through all reals below $x$ in $<_{L}$ for a real $z<_{L} x$ such that $Q_{A}(z) \downarrow=1$. If such a real is found, then $x \neq a$. Otherwise, $x=a$.

We will need the following result of Jensen and Karp:

Theorem 11. Let $\alpha \in O n$ be a limit of admissibles, and let $\phi$ be a $\Sigma_{1}$-statement. Then $\phi$ is absolute between $L_{\alpha}$ and $V_{\alpha}$.

Proof. See [JeKa].

\section{UnReCOGNizABles EveryWhere}

The goal of this section is to show the result announced above: If $\gamma>\omega_{\omega}^{C K}$ is such that $L_{\gamma+1}-L_{\gamma}$ contains a real number, then it already contains a nonrecognizable real number. To show this, we will use Cohen-forcing over models of $K P$. A general reference for the forcing technique is $[\mathrm{Ku}]$. For forcing over set theories weaker than $Z F C$, we refer the reader to [Sa2], [Ch] and [Ma].

Definition 12. An ordinal $\gamma$ is called an index iff $L_{\gamma+1}-L_{\gamma}$ contains a subset of $\omega$.

Theorem 13. Let $\mathbb{P} \in L_{\xi}$ be a notion of forcing, where $\xi$ is indecomposable, let $\gamma>\xi$ be admissible and let $G$ be $\mathbb{P}$-generic over $L_{\gamma}$. Then $L_{\xi}[G]$ in the sense of relative constructibility coincides with $L_{\xi}[G]$ in the sense of generic extensions.

Proof. This follows from the proof of Proposition 9.5 of [Ma].

Theorem 14. Let $x \in R E C O G$. Then $x \in L_{\omega_{\omega}^{C K, x}}$. Consequently, if $x$ is recognizable, but not ITRM-computable, we have $\omega_{\omega}^{C K, x}>\omega_{\omega}^{C K}$.

Proof. Let $P$ be a program that recognizes $x$.

Then $L_{\omega_{\omega}^{C K, x}}[x] \models \exists y P^{y} \downarrow=1$. Now $\phi \equiv \exists y P^{y} \downarrow=1$ is a (set theoretical) $\Sigma_{1}$-statement, stating that there are a real $y$ and a set $c$ such that $c$ codes the $P$-computation in the oracle $y$ and ends with 1 . By JensenKarp (see Theorem 11), this is absolute between $V_{\alpha}$ and $L_{\alpha}$ whenever 
$\alpha$ is a limit of admissibles. Now $\omega_{\omega}^{C K, x}=\sup \left\{\omega_{i}^{C K, x} \mid i \in \omega\right\}$ is a limit of admissibles, so $\phi$ is absolute between $V_{\alpha}$ and $L_{\omega_{\omega}^{C K, x}}$. Also, as $\phi$ is $\Sigma_{1}$, it is upwards absolute. Hence $L_{\omega_{\omega}^{C K, x}}[x] \models \phi \Longrightarrow V_{\omega_{\omega}^{C K, x}} \models \phi \Longrightarrow$ $L_{\omega_{\omega}^{C K, x}} \Longrightarrow L_{\omega_{\omega}^{C K, x}}[x]$, so $\phi$ is absolute between $L_{\omega_{\omega}^{C K, x}}[x]$ and $L_{\omega_{\omega}^{C K, x}}$. As $\phi$ holds in $L_{\omega_{\omega}^{C K, x}}[x]$, it follows that $\phi$ holds in $L_{\omega_{\omega}^{C K, x}}$. So $L_{\omega_{\omega}^{C K, x}}$ contains a real $r$ such that $P^{r} \downarrow=1$. By absoluteness of computations, $P^{r} \downarrow=1$ also holds in $V$. So $P^{r} \downarrow=1$. As $P$ recognizes $x$, it follows that $x=r$. Hence $x \in L_{\omega_{\omega}^{C K, x}}$.

Now let $x$ be recognizable, but not computable. As $x$ is not computable, we have $x \notin L_{\omega_{\omega}^{C K}}$. By the first part of the claim, $x \in L_{\omega_{\omega}^{C K, x}}$. Hence $\omega_{\omega}^{C K, x}>\omega_{\omega}^{C K}$.

This immediately leads to the following dichotomy:

Corollary 15. If $x \subseteq \omega$ is such that $\omega_{\omega}^{C K, x}=\omega_{\omega}^{C K}$, then either $x$ is ITRM-computable or $x$ is not ITRM-recognizable.

Proof. If $x$ is ITRM-computable, then $x$ is clearly ITRM-recognizable (see [Ca]). If $x$ is not ITRM-computable, then $x \notin L_{\omega_{\omega}^{C K}}$, hence $x \notin$ $L_{\omega_{\omega}^{C K, x}}$ if $\omega_{\omega}^{C K, x}=\omega_{\omega}^{C K}$. By Theorem 14 then, $x$ is not recognizable.

For the next lemma, we need the following result of Mathias:

Theorem 16. If $M$ is admissible, $\mathbb{P} \in M$ and $G$ is an $(M, \mathbb{P})$-generic filter meeting each dense open subclass of $M$ that is the union of a $\Sigma_{1}(M)$ and a $\Pi_{1}(M)$ class, then $M^{\mathbb{P}}[G]$ is admissible.

Proof. This is Theorem 10.11 of [Ma].

Lemma 17. Let $\alpha$ be admissible, $(P, \leq) \in L_{\alpha}$ be a notion of forcing and $G$ be a filter on $P$ such that $P \cap D \neq \emptyset$ for every dense subset $D$ of $P$ such that $D \in L_{\alpha+1}$. Then $L_{\alpha}[G]$ is admissible.

Proof. This follows from Theorem 16, since unions of $\Sigma_{1}\left(L_{\alpha}\right)$ and $\Pi_{1}\left(L_{\alpha}\right)$ definable subsets of $P$ are clearly elements of $L_{\alpha+1}$.

Corollary 18. Let $\gamma \geq \omega_{\omega}^{C K}$, let $\left(P, \leq_{P}\right)$ be the notion of forcing for adding a Cohen real (i.e. $P$ consists of the finite partial functions from $\omega$ to 2 and $x \leq_{P} y$ iff $\left.y \subseteq x\right)$ and let $G$ be a filter on $(P, \leq)$ which intersects every dense $D \subseteq P$ such that $D \in L_{\gamma}$. Then $L_{\omega_{i}^{C K}}[G]$ is admissible for every $i \in \omega$.

Proof. This is immediate from Lemma 17 as $L_{\gamma} \supseteq L_{\omega_{\omega}^{C K}} \supseteq L_{\omega_{i}^{C K}+1}$ for all $i \in \omega$.

The following will be used to show that, for each index $\gamma \geq \omega_{\omega}^{C K}$, $L_{\gamma+1}-L_{\gamma}$ contains an unrecognizable real. Recall that $\gamma \in O n$ is said to be an index iff $\left(L_{\gamma+1}-L_{\gamma}\right) \cap \mathfrak{P}(\omega) \neq \emptyset$.

Theorem 19. Let $\gamma \geq \omega_{\omega}^{C K}$ be an index. Then there exists $x \in L_{\gamma+1}-L_{\gamma}$ with $\omega_{i}^{C K, x}=\omega_{i}^{C K}$ for all $i \in \omega$. In particular, this implies that $\omega_{\omega}^{C K, x}=\omega_{\omega}^{C K}$. 
Proof. Let $P$ be the notion of forcing for adding a Cohen real (see above). Let $G$ be an $L_{\gamma}$-generic filter on $P$ (i.e. $G$ intersects every dense subset of $P$ which lies inside $L_{\gamma}$ ). By Corollary $18, L_{\omega_{i}^{C K}}[G]$ is then admissible for all $i \in \omega$.

Now let $x:=\bigcup G \in \mathfrak{P}(\omega)$. We show that $L_{\omega_{i}^{C K}}[G]=L_{\omega_{i}^{C K}}[x]$ : As $x=\bigcup G \in L_{\omega_{i}^{C K}}[G]$, we have $L_{\omega_{i}^{C K}}[x] \subseteq L_{\omega_{i}^{C K}}[G]$ and $G \in L_{\omega_{i}^{C K}}[x]$ (since $G$ is definable from $x$ ), hence also $L_{\omega_{i}^{C K}}[G] \subseteq L_{\omega_{i}^{C K}}[x]$. More generally, if $\alpha$ is additively indecomposable (which certainly holds for $\alpha$ admissible) and if we have $x \in L_{\alpha}[y]$ and $y \in L_{\alpha}[x]$, then $L_{\alpha}[x]=L_{\alpha}[y]$. To see this, let $z \in L_{\beta}[x](\beta<\alpha)$ and $x \in L_{\gamma}[y](\gamma<\alpha)$. Then $z \in L_{\beta}[x] \in L_{\gamma+\beta+1}[y] \subseteq L_{\alpha}[y]$, hence $L_{\alpha}[x] \subseteq L_{\alpha}[y] . L_{\alpha}[y] \subseteq L_{\alpha}[x]$ now follows by symmetry.

Now it follows that $L_{\omega_{1}^{C K}}[x] \models K P$, i.e. $\omega_{1}^{C K}$ is $x$-admissible, so that $\omega_{1}^{C K} \geq \omega_{1}^{C K, x} \geq \omega_{1}^{C K}$. Consequently, we get $\omega_{1}^{C K, x}=\omega_{1}^{C K}$. Now assume inductively that $\omega_{i}^{C K}=\omega_{i}^{C K, x}$ for some $i \in \omega$. It then follows that $L_{\omega_{i+1}^{C K}}[x] \models K P$, hence $\omega_{i+1}^{C K}$ is $x$-admissible and thus $\omega_{i+1}^{C K}>\omega_{i}^{C K, x}=\omega_{i}^{C K}$. But then $\omega_{i+1}^{C K} \geq \omega_{i+1}^{C K, x} \geq \omega_{i+1}^{C K}$, so $\omega_{i+1}^{C K, x}=\omega_{i+1}^{C K}$. This now gives us $\omega_{i}^{C K, x}=\omega_{i}^{C K}$ for all $i \in \omega$, so that $\omega_{\omega}^{C K, x}=\omega_{\omega}^{C K}$. Next, we demonstrate that $G$ - and hence $x=\bigcup G$ - is definable over $L_{\gamma}$ and hence elements of $L_{\gamma+1}$. This can be seen as follows: As $\gamma$ is an index, there is $f: \omega \rightarrow L_{\gamma}$ surjective such that $f \in L_{\gamma+1}$ and hence definable over $L_{\gamma}$ Now define $g:\left({ }^{<\omega} 2, \omega\right) \rightarrow^{<\omega} 2$ thus: Let $g(\vec{s}, i)$ be the lexically minimal element of $f(i)$, of which $\vec{s}$ is a subsequence if $f(i) \subseteq^{<\omega} 2$ is a dense subset of $P$ otherwise let $g(\vec{s}, i)=\vec{s}$. Now, define recursively: $h(0)=\emptyset, h(i+1)=g(h(i), i+1)$. This recursion can be carried out definably over $L_{\gamma}$ as follows: Set (for $\left.i \in \omega\right) h(i)=x$ iff $(i=0 \wedge x=\emptyset) \vee\left(i \geq 1 \wedge \exists\left(\overrightarrow{s_{0}}, \ldots, \overrightarrow{s_{i-1}}\right)\left[\forall j \in i\left(\left(j=0 \wedge \overrightarrow{s_{0}}=\emptyset\right) \vee\left(\overrightarrow{s_{j}}=\right.\right.\right.\right.$ $\left.\left.\left.\left.g\left(\overrightarrow{s_{j-1}}, j\right)\right)\right) \wedge x=g\left(\overrightarrow{s_{i-1}}, i\right)\right]\right)$. This is definable over $L_{\gamma}$, as $g$ is definable over $L_{\gamma}$ and all finite sequences of elements of $P$ are contained in $L_{\omega}$, and hence certainly in $L_{\omega_{\omega}^{C K}}$. It follows that $x \in L_{\gamma+1}$. That $\omega_{i}^{C K, x}=\omega_{i}^{C K}$ holds for all $i \in \omega$ was seen above.

Finally, we show that $x \notin L_{\gamma}$ : Roughly, this follows immediately from the fact that $G$ is definable from $x$ and that $G$ is generic over $L_{\gamma}$ as in the case of Cohen-forcing for $Z F C$ models. More precisely, let $z \in \mathfrak{P}(\omega) \cap L_{\gamma}$. Also, let $\beta<\gamma$ be minimal such that $z \in L_{\beta+1}-L_{\beta}$. Then $D_{z}:=\left\{b \in^{<\omega} 2 \mid \exists i \in \omega b(i) \neq z(i)\right\}$ is dense in $P$ and definable over $L_{\beta}$, hence an element of $L_{\gamma}$. Consequently, every $D_{z}$ has nonemptys intersection with every $L_{\gamma}$-generic filter $G$, so that $\bigcup G \neq z$. As this holds for all $z \in L_{\gamma}$, we get $x=\bigcup G \notin L_{\gamma}$.

We can now show that new unrecognizables appear wherever possible, i.e. are generated at every index stage: 
Theorem 20. Let $\gamma \geq \omega_{\omega}^{C K}$ be an index. Then $L_{\gamma+1}-L_{\gamma}$ contains an unrecognizable real.

Proof. By Theorem 19, $L_{\gamma+1}-L_{\gamma}$ contains a real $x$ such that $\omega_{\omega}^{C K, x}=$ $\omega_{\omega}^{C k}$. By Corollary 15, $x$ is not recognizable.

\section{The halting nUmber is ReCognizable}

We obtain a very natural lost melody by showing that the halting number for $I T R M \mathrm{~s}$ is in fact recognizable. Fix a natural well-ordering $\left(P_{i} \mid i \in \omega\right\}$ of the ITRM-programs in order type $\omega$ by e.g. sorting the programs lexicographically. This real $h$ is natural insofar its definition is purely internal to $I T R M \mathrm{~s}$ (e.g. not in any way related to $L$ ) and it is also arguably the first non-computable real coming to mind.

We start by showing that, given $h$, there is a universal ITRM:

Lemma 21. There is an ITRM-program $P$ such that, for every $(i, j) \in$ $\omega^{2}$, we have $P^{h}(p(i, j)) \downarrow=k+1$ if $P_{i}(j) \downarrow=k$ and $P^{h}(p(i, j)) \downarrow=0$ if $P_{i}(j) \uparrow$. That is, $P$ can, given $i$, compute the function computed by $P_{i}$.

Proof. $P$ works as follows: Given $i$ and $j$, first use $h$ to check whether $P_{i}(j) \downarrow$. If $P_{i}(j) \uparrow, P$ returns 0 . Otherwise, we carry out the following procedure for each $k \in \omega$ : Compute (which can be done with a standard register machine, in fact) an index $l=l(i, j, k)$ such that $P_{l} \downarrow$ iff $P_{i}(j) \downarrow=k$. $P_{l}$ will use a halting problem solver for $P_{i}$ (which can be easily obtained from $P_{i}$ ), i.e. a sub-program $Q$ such that $Q(j) \downarrow=1$ iff $P_{i}(j) \downarrow$ and $Q(j) \downarrow=0$, otherwise. If it turns out that $Q(j)=0$, then $P_{l}$ enters an infinite loop. Otherwise, we wait until $P_{i}(j)$ has stopped and check whether the outcome is $k$. If it is, we stop, otherwise we enter an infinite loop. (Note that $P$ is not required to do all this; it is only required that $P$ can compute a code for a program that does this, which is in fact easy).

Using $l$ and $h$, we can easily check whether $P_{i}(j) \downarrow=k$. If so, we return $k+1$. Otherwise, we continue with $k+1$.

As $P_{i}(j) \downarrow$ is already clear at this point, this has to lead to the value of $P_{i}(j)$ after finitely many iterations.

The next step is that, using $h$, a code for $L_{\omega_{i}^{C K}}$ can be computed uniformly in $i$.

Corollary 22. There is an ITRM-program $Q$ such that, for every $i \in \omega, Q^{h}(i)$ computes a code for $L_{\omega_{i}^{C K}}$. (I.e.: $Q^{h}(n)$ halts for every $n \in \omega$ and $\left\{j \in \omega \mid Q^{h}(p(i, j) \downarrow=1\}\right.$ will be a code for $L_{\left.\omega_{i}^{C K} \text {. }\right)}$

Proof. First note that codes for $L_{\omega_{i}^{C K}}$ are uniformly recognizable in $i$, i.e. there is a program $R$ such that, for every $i \in \omega, x \subseteq \omega, R^{x}(i) \downarrow=1$ iff $x$ codes $L_{\omega_{i}^{C K}}$ and otherwise $R^{x}(i) \downarrow=0$. This can be obtained using 
the well-foundedness checker combined with the first-order checker described in [ITRM] for $V=L+K P+$ 'There are exactly $i-1$ admissible ordinals'.

Using $h$, we can now run through $\omega$, first testing whether $P_{k}(j)$ will halt for each $j \in \omega$ and then, using $P$ from the last lemma, whether $P_{k}$ will compute a code for $L_{\omega_{i}^{C K}}$. (We can evaluate $P_{k}(j)$ for every $j$ using $P$ from the last lemma and then use $R$ to recognize whether the computed number is a code.)

As $L_{\omega_{i}^{C K}}$ has ITRM-computable codes, the minimal index $l$ such that $P_{l}$ computes a code for $L_{\omega_{i}^{C K}}$ will eventually be found in this way.

After that, we can, again using $P$ from the last lemma, evaluate $l$ to compute the desired code.

These bits can now be put together to form a code for $L_{\omega_{\omega}^{C K}}$. This code will be a bit different from the codes considered so far, as we allow one element of the coded structure to be represented by arbitrary many elements of $\omega$.

Definition 23. Let $(X, \in)$ be a transitive $\in$-structure. Furthermore, let $f: \omega \rightarrow X$ be surjective. Then $\{p(i, j) \mid f(i) \in f(j)\}$ is called an odd code for $(X, \in)$.

Odd codes can be evaluated in the same way that the codes we used so far could. The possibility of elements appearing repeatedly hinders none of those methods. It is helpful, however, to note that the equality is computable:

Proposition 24. There is an ITRM-program $\bar{T}$ such that, for every odd code $x$ for a well-founded $\in$-structure $(X, \in)$ (with associated function $f: \omega \rightarrow X)$ and all $i, j \in \omega, \bar{T}^{x}(p(i, j)) \downarrow=1$ iff $f(i)=f(j)$ and $\bar{T}^{x} \downarrow=0$, otherwise.

Furthermore, there is an ITRM-program $T$ such that, for every two odd codes $x$ and $y$ for well-founded $\in$-structures $(X, \in)$ and $(Y, \in)$ (with associated functions $f_{1}$ and $\left.f_{2}\right), T^{x \oplus y}(p(i, j)) \downarrow=1$ iff $f_{1}(i)=f_{2}(j)$ and $T^{x \oplus y}(p(i, j)) \downarrow=0$, otherwise.

Finally, there is an ITRM-program $T_{\in}$ such that, for every two odd codes $x$ and $y$ for well-founded $\in$-structures $(X, \in)$ and $(Y, \in)$ (with associated functions $f_{1}$ and $\left.f_{2}\right), T_{\in}^{x \oplus y}(p(i, j)) \downarrow=1$ iff $f_{1}(i) \in f_{2}(j)$ and $T_{\in}^{x \oplus y}(p(i, j)) \downarrow=0$, otherwise.

Proof. This is an easy application of the techniques developed in [ITRM]. We give a brief impression how this works: To decide, given $x, y \subseteq \omega$ coding transitive $\in$-structures and $i, j \in \omega$, whether $i$ and $j$ represent the same element, we use a stack. Initially, the stack contains $p(i, j)$. We then need to decide whether $f_{1}(i) \subseteq f_{2}(j)$ and $f_{2}(j) \subseteq f_{1}(i)$. To see the former, we use $x$ to successively consider the natural numbers coding elements of $f_{1}(i)$. For each such natural number $n$, we search, 
using $y$, through all natural numbers coding elements of $f_{2}(j)$. For each such element $m$, we put $p(n, m)$ on our stack and decide whether $f_{1}(n)=f_{2}(m)$. When such an $m$ is found, we replace the content of the stack register with $p(i, j)$ (this ensures that the liminfs will be as desired). When all $m$ have been tried and no $m$ with $f_{2}(m)=f_{1}(n)$ has been found, then $f_{1}(i) \neq f_{2}(j)$. Otherwise, we continue with the next $n$. When an $m$ with $f_{2}(m)=f_{1}(n)$ has been found for every such $n$, we have $f_{1}(i)=f_{2}(j)$. That the algorithm must terminate follows from the well-foundedness of $(X, \in)$ and $(Y, \in)$.

Lemma 25. There is an ITRM-program $S$ such that $S^{h}$ computes an odd code $c$ for $L_{\omega_{\omega} K}$.

Proof. The idea is to reserve $\omega$ bits for coding $L_{\omega_{i}^{C K}}$; in one portion (the $i$-th portion), we use $Q^{h}$ to compute a code $c_{i}$ for $L_{\omega_{i}^{C K}}$ with corresponding surjection $f_{i}$. Then we use $T$ from the last proposition to relate the portions.

More precisely, we construct $c$ as follows: Fist, for all $i, j, k \in \omega$, we let $p(p(j, i), p(k, i)) \in x$ iff $p(j, k) \in c_{i}$. It remains to decide the bits of the form $p\left(p\left(j, i_{1}\right), p\left(k, i_{2}\right)\right)$ with $i_{1} \neq i_{2}$. This corresponds to the question whether $f_{i_{1}}(j) \in f_{i_{2}}(k)$, which can be answered using $T_{\in}$ from Proposition 24.

Theorem 26. Let $h:=\left\{i \in \omega \mid P_{i} \downarrow\right\}$ be the set of indices of halting ITRM-programs. Then $h \in R E C O G$.

Proof. Let $x$ be the real in the oracle. We can check, using Lemma 5, whether $S^{x}$ computes an odd code $c$ for $L_{\omega_{\omega}^{C K}}$. If not, return 0 .

Checking whether certain programs halt amounts to checking whether certain first-order statements (expressing that $P_{i}$ halts, i.e. there is a set coding a halting computation according to $P$ ) hold in $L_{\omega_{\omega}^{C K}}$, which can be done using $c$ by Lemma 6 . We can then compare the results of the computation with the oracle number $x$. If the agree, then $x=h$, otherwise $x \neq h$. This identifies $h$.

\section{Potential Recognizability}

We saw above (via Jensen-Karp, see Theorem 14) that $x \in R E C O G$ implies that $x \in L_{\omega_{\omega}^{C K, x}}$. Reals without this property are hence ruled out, we concentrate on those that have it.

Definition 27. $x \subseteq \omega$ is potentially recognizable iff $x \in L_{\omega_{\omega}^{C K, x}}$. We denote the set of potentially recognizable reals by PRECOG.

Theorem 28. Let $\gamma$ be an index. Then either all potentially recognizable elements of $L_{\gamma+1}-L_{\gamma}$ are recognizable or none is. 
Proof. Suppose $a \in\left(L_{\gamma+1}-L_{\gamma}\right) \cap R E C O G$ and $x \in\left(L_{\gamma+1}-L_{\gamma}\right) \cap P R E C O G$. We want to show that $x \in R E C O G$. Pick a program $Q$ that recognizes $a$. As $x \in P R E C O G$, there is $i \in \omega$ such that $x \in L_{\omega_{i}^{C K, x}}$. In particular, we have $L_{\gamma+1} \in L_{\omega_{i}^{C K, x}}$. Hence $c=c c\left(L_{\gamma+1}\right)$, the $<_{L}$-minimal real code for $L_{\gamma+1}$ is computable from $x$. Let $P$ be a program that computes $c$ from $x$.

To identify whether $y=x$ (with $y$ in the oracle), we first use a halting problem solver (see 3) for $P$ to check whether $P^{y}(i) \downarrow$ for all $i \in \omega$. If not, then $y \neq x$. If yes, we check whether $P^{y}$ computes a code $d$ for an $L$-level containing $y$. If not, then $y \neq x$. If yes, we check, as in the proof of the Lost Melody Theorem in [ITRM], whether $d$ is $<_{L}$-minimal with that property (this is possible as $x \in L_{\gamma+1}$ ). If not, then $y \neq x$. If yes, we check whether the structure coded by $d$ contains a real $r$ such that $Q^{r} \downarrow=1$. This can be done Lemma 5. If there is no such $r$, then $y \neq x$. If there is, we check whether the structure coded by $d$ contains an $L$-level that also contains $r$ (this checks the minimality of $\gamma$ ). If so, then $y \neq x$. Otherwise, we know that $P^{y}$ computes $c$. But in $c$, $x$ is coded by some fixed natural number $n$ which can be given to the program in advance. All that remains is hence to check whether $y$ is the number coded by $n$ in $c$. If not, then $y \neq x$, otherwise $y=x$. So this procedure recognizes $x$, hence $x \in R E C O G$.

This allows us to give a characterization of recognizability in purely set-theoretical vocabulary, without reference to machines:

Theorem 29. Let $x \in P R E C O G$. Then $x \in R E C O G$ iff there exists a $\Sigma_{1}$-formula $\phi$ of set theory without parameters such that $x$ is the unique witness for $\phi(v)$ in $L_{\omega_{\omega}^{C K, x}}$.

Proof. If $x \in R E C O G$, then $x \in P R E C O G$ by Theorem 14 above. Now, if $P$ is a program that recognizes $x$, then $P^{x} \downarrow=1$ is $\Sigma_{1}$-expressable over $L_{\omega_{\omega}^{C K, x}}$. By upwards preservation of $\Sigma_{1}$-statements, if $L_{\omega_{\omega}^{C K, x}}=$ $P^{y} \downarrow=1$ for some $y \neq x$, then $P^{y} \downarrow=1$ in the real world, which contradicts the assumption that $P$ recognizes $x$.

On the other hand, if $x$ is definable as above, then let $L_{\gamma}$ be the first $L$ level containing $x$ such that $L_{\gamma}=\phi(x)$. Then $\gamma<\omega_{\omega}^{C K, x}$, so $c:=c c\left(L_{\gamma}\right)$ can be computed from $x$, say by program $Q$. Given a real $y$ and $c$ in the oracle, we can check whether $y \in L_{\gamma}$ and $L_{\gamma} \models \phi(y)$ holds.

Checking whether some oracle number $y$ is equal to $x$ then works as follows: Check (using Lemma 5 and the techniques from the proof of the Lost Melody theorem in [ITRM]) whether $Q^{y}$ computes a minimal code for an $L$-level containing $y$, then check whether $\phi(y)$ holds in that $L$-level and then whether it fails in all earlier $L$-levels. If all of this holds, then $y=x$ (since $\Sigma_{1}$ is preserved upwards), otherwise $y \neq x$. 
The same argument can be adapted to characterize IT RM-decidable sets of reals (a set $A \subseteq \mathfrak{P}(\omega)$ is ITRM-decidable iff there is an ITRMprogram $P$ such that $P^{x} \downarrow=1$ iff $x \in A$ and $P^{x} \downarrow=0$, otherwise, for all $x \in \mathfrak{P}(\omega))$. ITRM-decidable sets of reals were considered and demonstrated to be $\Delta_{2}^{1}$ in [KoMi].

Theorem 30. Let $A \subseteq \mathfrak{P}(\omega)$. Then $A$ is ITRM-decidable iff there exist $n \in \omega$ and a parameter-free $\Sigma_{1}$-formula $\phi(y)$ with a single free variable $y$ such that $A=\left\{x \in \mathfrak{P}(\omega) \mid L_{\omega_{n}^{C K, x}}[x] \models \phi(x)\right.$.

Proof. Assume first that $A$ is ITRM-decidable. Let $P$ be an ITRMprogram that decides $A$. The computation is contained in $L_{\omega_{n}^{C K, x}}[x]$ if $n$ is larger than the number of registers used by $P$. Hence membership of $x$ in $A$ can be expressed as the existence of a computation by $P$ with input $x$ that terminates with output 1 , which is $\Sigma_{1}$ over $L_{\omega_{n}^{C K, x}}[x]$.

On the other hand, if $x \in A$ is characterized as described in the assumptions by a $\Sigma_{1}$-formula over $L_{\omega_{n}^{C K, x}}[x]$, we can $I T R M$-decide $A$ by computing a code for $L_{\omega_{n}^{C K, x}}[x]$ from $x$, then identifying the natural number representing $x$ in this code and finally using the code for evaluating $\phi$ in $L_{\omega_{n}^{C K, x}}[x]$. The only new step here is the computation of

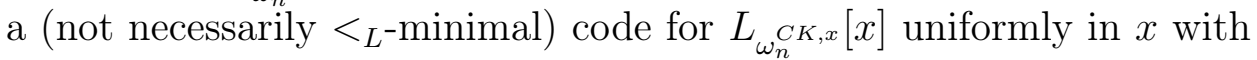
a program that always halts. Using a halting problem solver $H$ as in Theorem 3 for ITRMs with $n+3$ registers, this can be done as follows: Given $x$ in the oracle and taking $\left(P_{j} \mid j \in \omega\right)$ to be a natural enumeration of the ITRM-programs using at most $n+3$ registers, we perform the following routine for every $i \in \omega$ : First, check whether $P_{i}^{x}$ computes a real, i.e. whether $P_{i}^{x}(k)$ halts for every $k \in \omega$. This can be done using $H$. If this is not the case, restart the routine with $i+1$. Otherwise, check, with the usual techniques, whether the real $r$ computed by $P_{i}^{x}$ is a code for $L_{\omega_{n}^{C K, x}}[x]$ (i.e. an admissible level of the $L[x]$-hierarchy containing exactly $n-1 x$-admissible ordinals). If not, restart the routine with $i+1$. Otherwise, an algorithm computing the desired code is found.

Theorem 31. For all $x \subseteq \omega, x$ is recognizable iff $x \in L_{\omega_{\omega}^{C K, x}}$ and $L_{\omega_{\omega}^{C K, x}} \models R E C O G(x)$. In particular, if $L_{\alpha} \models Z F^{-}$and $x \in \mathfrak{P}(\omega) \cap L_{\alpha}$, then $x \in R E C O G$ holds iff $L_{\alpha}=R E C O G(x)$.

Proof. Suppose first that $x \in R E C O G$, and let $P$ be a program that recognizes $x$. Then $x \in L_{\omega_{\omega}^{C K, x}}$ by Theorem 14. By [Ch], if $z \in L_{\gamma}$ and $\gamma^{+}$is the smallest admissible ordinal greater than $\gamma$, then $\omega_{1}^{C K, z} \leq \gamma^{+}$. Inductively, we get that $\omega_{i}^{C K, z} \leq \gamma^{+i}$, where $\gamma^{+i}$ is the $i$ th admissible ordinal above $\gamma$. Inductively, it follows that $\omega_{\omega}^{C K, z} \leq \omega_{\omega}^{C K, x}$ for all $z \in L_{\omega_{\omega}^{C K, x}}$ when $x$ is such that $x \in L_{\omega_{\omega}^{C K, x}}$. This implies that $P^{z}$ stops after at most $\omega_{\omega}^{C K, x}$ many steps for all $z \in L_{\omega_{\omega}^{C K, x}}$ and hence that $P^{z}$ can be carried out inside $L_{\omega_{\omega}^{C K, x}}$ for all $z \in{\stackrel{\omega}{\omega_{\omega}}}_{\omega K, x}$. Hence, since $P$ 
recognizes $x$, we have $L_{\omega_{\omega}^{C K, x}} \models P^{z} \downarrow=0$ for all $z \neq x$ and furthermore $L_{\omega_{\omega}^{C K, x}} \models P^{x} \downarrow=1$. Hence $L_{\omega_{\omega}^{C K, x}} \models R E C O G(x)$.

On the other hand, assume that $x \in L_{\omega_{\omega}^{C K, x}}$ and that

$L_{\omega_{\omega}^{C K, x}} \models \operatorname{RECOG}(x)$. Hence $P^{x} \downarrow=1$ and $P^{z} \downarrow=0$ for all $z<_{L} x$. Now let $Q$ be a program such that $Q^{x}$ computes the $<_{L}$-minimal code of the first $L$-level containing $x$. Then $x$ can be recognized as follows: Given some real $r$ in the oracle, first check, using Lemma 5, whether $P^{r} \downarrow=1$. If not, then $r \neq x$. Otherwise check - applying Lemma 5 to $Q$ - whether $Q^{r}(i) \downarrow$ for all $i \in \omega$. If not, then $r \neq x$. If yes, check whether $Q^{r}$ codes a minimal $L$-level containing $r$. If not, then $r \neq x$. If yes, check whether $Q^{r}$ is $<_{L}$-minimal with this property, using the usual strategy. If not, then $r \neq x$. Otherwise, use $Q^{r}$ (and the halting problem solver for $P$ ) to check whether there is any real $y<_{L} x$ such that $P^{y} \downarrow=1$. If that is the case, then $r \neq x$. If it isn't, then $r$ is $<_{L}$-minimal with $P^{r} \downarrow=1$ and hence $r=x$.

\section{MORE ON GAPS}

Definition 32. (See [Ca]) A strong substantial gap is an ordinal interval $[\alpha, \beta]$ with $\beta>\alpha$ such that every $\gamma \in[\alpha, \beta]$ is an index and such that $L_{\beta}-L_{\alpha}$ contains no recognizables, while $L_{\beta+1}-L_{\beta}$ and $L_{\alpha}-L_{\gamma}$ do contain recognizables for every $\gamma<\alpha$. A weak substantial gap is an ordinal interval $[\alpha, \beta]$ such that $\alpha$ is an index, the set of indices in that interval is unbounded in $\beta$ and such that $L_{\beta+1}-L_{\alpha}$ contains no recognizables.

Note that it is shown in [Ca] that strong substantial gaps exist (it suffices to see that there is some index $\gamma \in O n$ with $\left.\left(L_{\gamma+1}-L_{\gamma}\right) \cap R E C O G=\emptyset\right)$. We can now show that gaps in the recognizables must be infinite. The same reasoning in fact supports much stronger conclusions, as we shall presently see. A minor technical issue arises in the following arguments due to the fact that we consider levels of the $L$-hierarchy with only very weak closure properties, so that we cannot exclude the possibility that some real coding such a level (or its ordinal height) will actually be contained in it as an element, while our arguments need codes arising only at a later stage. We hence make the following definition:

Definition 33. If $\gamma<\omega_{1}$, we denote by $c c^{\prime}\left(L_{\gamma}\right)$ the $<_{L}$-minimal real coding $L_{\gamma}$ which is not an element of $L_{\gamma}$.

Theorem 34. There are no strong substantial gaps of finite length. Furthermore, strong gaps always start with limit ordinals.

Proof. Assume for a contradiction that there is a strong substantial gap of length $i$, where $i \in \omega$. Let $\alpha \in O n$ be minimal such that $[\alpha, \alpha+i]$ is a strong substantial gap of length $i$. It is easy to see that $c c\left(L_{\alpha+i}\right)$ is recognizable by the usual arguments: Given $x$, check 
whether $x$ codes an $L$-level at which a strong substantial gap of length $i$ ends. This can be done by Lemma 6 . The minimality of $x$ can then be checked by the routines for evaluating truth predicates described in the last section of [ITRM]. there. By the results on the computational strength of $I T R M \mathrm{~s}$, one readily obtains that from the $<_{L}$-minimal code $c$ of $L_{\alpha}$ which is not an element of $L_{\alpha}$, we can compute $c c\left(L_{\alpha+i}\right)$, say by program $P$. Note that, by the definition of a substantial strong gap. we will have $c c\left(L_{\alpha+i}\right) \in L_{\alpha+i+1}$. But this allows us to recognize $c$ : Given the oracle $x$, first check (applying Lemma 5 to $P$ ) whether $P^{x}$ computes $c c\left(L_{\alpha+i}\right)$ - which is possible as $c c\left(L_{\alpha+i}\right)$ is recognizable. Now, in $c c\left(L_{\alpha+i}\right), c$ is represented by some integer $j$. It hence only remains to see whether $x$ is the number represented by $j$ in $c c\left(L_{\alpha+i}\right)$, which is also easy to do.

This implies that $c$ is recognizable. But, by definition, $c \in L_{\alpha+1}-L_{\alpha}$. Hence $\left(L_{\alpha+i}-L_{\alpha}\right) \cap R E C O G \neq \emptyset$, which contradicts the assumption that $\alpha$ starts a gap.

To see that, if $\alpha$ starts a strong substantial gap, $\alpha$ has to be a limit ordinal, we proceed as follows: Assume for a contradiction that $\alpha$ starts a strong substantial gap and $\alpha=\beta+1$. Since $\alpha$ starts the gap, $L_{\alpha}-$ $L_{\beta}$ contains a recognizable real $r$. We argue that $c^{\prime}\left(L_{\alpha}\right) \in L_{\alpha+1}-$ $L_{\alpha}$ is recognizable, which contradicts the assumption that $\alpha$ starts a gap. A procedure for recognizing $c c^{\prime}\left(L_{\alpha}\right)$ works as follows: Given $x$, simply check whether $x$ is the $<_{L}$-minimal code of a minimal $L$-level containing $r$ which is not contained in that level. This is possible since $r$ is recognizable.

Theorem 35. If $\alpha$ starts a weak substantial gap $[\alpha, \beta]$, then $\beta \geq \omega_{\omega}^{C K, c c^{\prime}(\alpha)}$.

Proof. Assume that $\alpha$ starts a weak substantial gap $[\alpha, \beta]$ where $\beta<$ $\omega_{\omega}^{C K, c c^{\prime}(\alpha)}$, so that $\beta<\omega_{i}^{C K, c c^{\prime}(\alpha)}$ for some minimal $i \in \omega$. By definition, $\alpha$ is an index, so that $c c^{\prime}(\alpha) \in L_{\alpha+1}$. By definition of $c c^{\prime}$, we may assume that $c c^{\prime}(\alpha) \notin L_{\alpha}$. We now want to argue that $c c^{\prime}(\alpha) \in R E C O G$, which will be a contradiction to the assumption that $\alpha$ starts a gap. From $c c^{\prime}(\alpha)$, one can compute $c c^{\prime}\left(L_{\omega_{i}^{C K, c c^{\prime}(\alpha)}}\right)$ by Theorem 9 . Let $P^{\prime}$ be an ITRM-program computing $c c^{\prime}\left(L_{\omega_{i}^{C K, c c(\alpha)}}\right)$ in the oracle $c c^{\prime}(\alpha)$. Since $i \in \omega$ is a fixed natural number, we can use $i$ together with $P^{\prime}$ to determine, for an arbitrary oracle $x$, whether $P^{\prime x}$ computes a $<_{L^{-}}$minimal code for $L_{\omega_{i}^{C K, x}}$. We can hence also compute the $<_{L^{-}}$ minimal code for $L_{\beta+1}$ in the oracle $c c^{\prime}(\alpha)$, using program $P$, say. By our assumption that $\beta$ ends the gap, we must have $R E C O G \cap\left(L_{\beta+1}-\right.$ $\left.L_{\beta}\right) \neq \emptyset$; say $r \in R E C O G \cap\left(L_{\beta+1}-L_{\beta}\right)$, and let $Q$ be a program for recognizing $r$. Now, given $x$ in the oracle, we can determine whether $P^{x}$ computes the minimal code for an $L$-level containing a real $z$ such 
that $Q^{z} \downarrow=1$. (This can be achieved by searching through the coded structure; since $r$ is recognized by $Q$, the calculation $Q^{z}$ will terminate for all reals $z$ from the coded structure.) If this is not the case, then $x \neq c c^{\prime}(\alpha)$. Otherwise, $P^{x}$ has computed $c c^{\prime}\left(L_{\beta+1}\right)$. In $c c^{\prime}\left(L_{\beta+1}\right)$, the real $c c^{\prime}(\alpha)$ is represented by some fixed natural number $k \in \omega$ (which can hence be given to our program). We can now simply test whether $x$ is the real coded by $k$ in $c c\left(L_{\beta+1}\right)$ by bitwise comparison. This allows us to recognize $c c^{\prime}(\alpha) \in L_{\alpha+1}-L_{\alpha}$, which contradicts the assumption that $\alpha$ starts a gap.

We now show that ordinals starting substantial gaps cannot be too easily definable.

Definition 36. $\alpha \in \omega_{1}$ is admissibly $\Sigma_{1}$-describable iff there exists a $\Sigma_{1}$-formula $\phi$ of set theory without parameters such that $\operatorname{cc}(\alpha)$ is

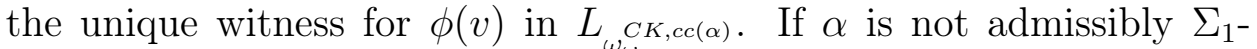
describable, we call it admissibly $\Sigma_{1}$-indescribable.

Theorem 37. Let $\alpha$ start a weak substantial gap. Then $\alpha$ is admissibly $\Sigma_{1}$-indescribable.

Proof. Assume for a contradiction that $\alpha$ is $\Sigma_{1}$-describable and starts a weak substantial gap. Then $\alpha$ is an index, so that $c c^{\prime}(\alpha) \in L_{\alpha+1}-L_{\alpha}$.

Now, if $\alpha$ was admissibly $\Sigma_{1}$-describable, we could compute from $c c^{\prime}(\alpha)$ the $<_{L}$-minimal code of the first $L_{\beta}$ containing a witness for some $\Sigma_{1}$-statement $\phi$ which characterizes $\alpha$. Let $P$ be a program that achieves this. By the usual procedure, we can check for an arbitrary oracle $x$ whether $P^{x}$ computes a minimal code of a minimal $L$-level containing such a witness. Now we must have $c c^{\prime}(\alpha) \in L_{\beta}$, so that $c c^{\prime}(\alpha)$ is represented in $c c^{\prime}\left(L_{\beta}\right)$ by some fixed natural number $k$. To determine whether $x=c c^{\prime}(\alpha)$, it hence only remains to check whether $x$ is equal to the number represented by $k$ in the structure coded by the real computed by $P^{x}$, which is also possible. So $c c^{\prime}(\alpha) \in L_{\alpha+1}-L_{\alpha}$ is recognizable, contradicting the assumption that $\alpha$ starts a gap.

In much the same way, we get:

Corollary 38. Let $[\alpha, \beta]$ be a strong substantial gap, and let $\gamma \in[\alpha, \beta]$. Then $\gamma$ is admissibly $\Sigma_{1}$-indescribable.

Proof. This follows by the same argument as above, since $\gamma$, being an element of a strong substantial gap, must be an index, which is the crucial property for this argument.

We have seen in Theorem 20 that, as soon as a new real appears in the $L$-hierarchy after $\omega_{\omega}^{C K}$ at all, a nonrecognizable real appears as well. In fact, reals appear that are not even potentially recognizable. This 
motivated us above to restrict our attention to potentially recognizable reals. Applying this here leads to the question whether at least the recognizability of potentially recognizable reals continues to hold for some stages beyond $L_{\omega_{\omega}^{C K}}$. This turns out to be true. In fact, the potentially recognizable reals continue being recognizable for quite a while after $L_{\omega_{\omega}^{C K}}$ :

Theorem 39. All elements of $L_{\omega_{\omega+\omega}^{C K}} \cap P R E C O G$ are recognizable.

Proof. This is clear for $x \in L_{\omega_{\omega}^{C K}}$. Hence let us assume from now on that $x \notin L_{\omega_{\omega}^{C K}}$. In the light of Theorem 28 (and the fact that, if $\gamma$ is an index, then an arithmetical copy of $L_{\gamma}$ appears in $L_{\gamma+1}$, see e.g. [Ch]), it suffices to show that, given $x \in L_{\omega_{\omega+\omega}^{C K}}$, the level $L_{\gamma}$ where $x$ appears has a recognizable $<_{L}$-minimal code $c c^{\prime}\left(L_{\gamma}\right)$ not in $L_{\gamma}$. For this, it suffices to show that, for all indices $\gamma$ between $\omega_{\omega}^{C K}$ and $\omega_{\omega+\omega}^{C K}, c c^{\prime}\left(L_{\gamma}\right)$ is recognizable. This can be seen as follows: Let $i \in \omega$ be minimal such that $\gamma \in L_{\omega_{\omega+i+1}^{C K}}$. Then $c c\left(L_{\omega_{\omega+i+1}^{C K}}\right)$ is ITRM-computable from $c c^{\prime}\left(L_{\gamma}\right)$, as, by our assumption, $\gamma \geq \omega_{\omega}^{C K}$, so that $\omega_{\omega}^{C K, c c\left(L_{\gamma}\right)} \geq \omega_{\omega+\omega}^{C K}-$ and $L_{\omega_{\omega+\omega}^{C K}}$ can easily be seen to contain $c c^{\prime}\left(L_{\omega_{\omega+i+1}^{C K}}\right)$. From now on, let $P$ be an ITRM-program that computes $c c\left(L_{\omega_{\omega+i+1}^{C K}}\right)$ from $c c^{\prime}\left(L_{\gamma}\right)$.

We claim that $c c\left(L_{\omega_{\omega+i+1}^{C K}}\right)$ is recognizable. This can be seen using the methods developed in the proof of the lost melody theorem in [ITRM] by first checking whether the oracle real $r$ codes an $L$-level, then, whether this level is admissible, contains a single limit of of admissibles and further $i$ admissibles greater than that limit and finally, whether $r$ is $<_{L}$-minimal with that property. (Note that the fixed natural number $i$ can be given to our program it advance.) Let $Q$ be a program that recognizes $c c\left(L_{\omega_{\omega+i+1}^{C K}}\right)$.

Now, $c c^{\prime}\left(L_{\gamma}\right)$, being an element of $L_{\omega_{\omega+i+1}^{C K}}$, is coded in $c c\left(L_{\omega_{\omega+i+1}^{C K}}\right)$ by some natural number $j$ (which can also be given to our program in advance). Hence, given $c c\left(L_{\omega_{\omega+i+1}^{C K}}\right)$, we can easily check whether some real $r$ is equal to $c c^{\prime}\left(L_{\gamma}\right)$ by checking whether it is equal to the element of $c c\left(L_{\omega_{\omega+i+1}^{C K}}\right)$ coded by $j$.

To recognize whether some real $r$ given in the oracle is equal to $c:=$ $c c^{\prime}\left(L_{\gamma}\right)$, we hence proceed as follows: First check, using Lemma 5, whether $P^{r}(k)$ halts with output 1 or 0 for all $k \in \omega$. If not, then $r \neq c$. Otherwise, $P^{r}$ computes some real $r^{\prime}$. Using $Q$, test whether or not $r^{\prime}=c c\left(L_{\omega_{\omega+i+1}^{C K}}\right)$. If not, then $r \neq c$. Otherwise, check whether $r$ is coded by $j$ in $r^{\prime}$. If not, then $r \neq c$, otherwise $r=c$.

This shows that, whenever $\omega_{\omega}^{C K} \leq \gamma<\omega_{\omega+\omega}^{C K}$ is an index, then $c c^{\prime}\left(L_{\gamma}\right)$ is a recognizable element of $L_{\gamma+1}-L_{\gamma}$. By our remark above, this shows that in fact all potentially recognizable reals in $L_{\omega_{\omega+\omega}^{C K}}-L_{\omega_{\omega}^{C K}}$ are recognizable. 


\section{Computability is not Decidable}

As a final application of the techniques developed in this paper, we prove that the set of ITRM-computable reals is not ITRM-decidable.

Theorem 40. There is no ITRM-program $P$ such that, for $L \ni x \subseteq \omega$, $P^{x} \downarrow=1$ iff $x$ is ITRM-computable and $P^{x} \downarrow=0$ otherwise.

Proof. Assume for a contradiction that $P$ is such a program, and let $P$ use $n$ registers. Hence, since $P^{x}$ halts for all $x \subseteq \omega$, it follows that $P^{x}$ halts in less than $\omega_{n+1}^{C K, x}$ many steps. Now, if $x$ is Cohen-generic over $L_{\omega_{n+1}^{C K}+1}$, then, by our results above, we have $\omega_{n+1}^{C K, x}=\omega_{n+1}^{C K}$.

It is easy to see that $L_{\omega_{n+2}^{C K}}$ contains such a real $x$. As $x \in L_{\omega_{\omega}^{C K}}, x$ is ITRM-computable, so that $P^{x} \downarrow=1$ in less than $\omega_{n+1}^{C K}$ many steps. Now $L_{\omega_{n+1}^{C K}}[x] \subseteq L_{\omega_{n+2}^{C K}}$, hence, as $L_{\omega_{n+2}^{C K}} \models P^{x} \downarrow=1$, it follows from the upwards absoluteness of $\Sigma_{1}$-formulas that $L_{\omega_{n+1}^{C K}}[x] \models P^{x} \downarrow=1$, where $x$ is the generic real. Hence, there is some finite subset $p$ of $x$ such that $p \Vdash P \cup \dot{G} \downarrow=1$, where $\dot{G}$ is the canonical name of the generic filter. Consequently, whenever $H \ni p$ is generic over $L_{\omega_{n+1}^{C K}+1}$ and $x=\bigcup H$, we have that $L_{\omega_{n+1}^{C K}}[x] \models P^{x} \downarrow=1$. Now let $x$ be Cohen-generic over $L_{\omega_{\omega}^{C K}}$ such that $p \subseteq x$. It is easy to see that such an $x$ exists. As $x \notin L_{\omega_{\omega}^{C K}}$, it is not ITRM-computable. However, we have $L_{\omega_{n+1}^{C K}}[x] \models P^{x} \downarrow=1$, so $P^{x} \downarrow=1$ by absoluteness of computations. Hence $P^{x} \downarrow=1$ for some non-computable $x$, a contradiction.

\section{ACKNOWLEDGMENTS}

We are indebted to Philipp Schlicht for helpful discussions on forcing over $K P$ and suggesting several very helpful references. We also thank Philip Welch for suggesting the use of Cohen-forcing over $L_{\omega_{\omega}^{C K}}$ as a method for obtaining unrecognizables low down in the constructible hierarchy.

\section{REFERENCES}

[Ba] J. Barwise. Admissible Sets and Structures. Springer (1975)

[Ca] M. Carl. The distribution of ITRM-recognizable reals. To appear in: Annals of Pure and Applied Logic, special issue for the CiE 2012

[Ch] C.T. Chong. A recursion-theoretic characterization of constructible reals. Bulletin of the London Mathematical Society 9, 241-244 (1977)

[Cu] N. Cutland. Computability. An introduction to recursive function theory. Cambridge University Press (1980)

[GHJ] V. Gitman, J.D. Hamkins, Th. A. Johnstone. What is the theory $Z F C$ without power set? arXiv:1110.2430 [math.LO]

[HaLe] J. Hamkins, A. Lewis. Infinite Time Turing Machines. Journal of Symbolic Logic 65(2), 567-604 (2000) 
[ITRM] M. Carl, T. Fischbach, P. Koepke, R. Miller, M. Nasfi, G. Weckbecker. The basic theory of infinite time register machines. Archive for Mathematical Logic 49 (2010) 2, 249-273

[JeKa] R. Jensen, C. Karp. Primitive Recursive Set Functions. In: Proceedings of Symposia in Pure Mathematics, Volume XIII, Part 1 (1971)

[KoMi] P. Koepke, Russell Miller. An enhanced theory of infinite time register machines. In Logic and Theory of Algorithms. A. Beckmann et al, eds., Lecture Notes in Computer Science 5028 (2008), 306-315

[KoWe] P. Koepke, P. Welch. A generalised dynamical system, infinite time register machines, and $\Pi_{1}^{1}-C A_{0}$. In CiE 2011. B. Löwe et al, eds., Lecture Notes in Computer Science 6735 (2011), 152-159.

[Ku] K. Kunen. Set Theory - An Introduction to Independence Proofs. Elsevier (1980)

[Ma] A.R.D. Mathias. Provident sets and rudimentary set forcing. Preprint. Available at https://www.dpmms.cam.ac.uk/ ardm/fifofields3.pdf

[Sa] G. Sacks. Higher recursion theory. Springer 1990.

[Sa2] G. Sacks. Countable admissible ordinals and hyperdegrees. Advances in Mathematics 19, 213-262 (1976)

[wITRM] P. Koepke. Infinite Time Register Machines. Logical Approaches to Computational Barriers, Arnold Beckmann et al., eds., Lecture Notes in Computer Science 3988 (2006), 257-266 